BRIEF

\title{
Hypertension Among US Adults by \\ Disability Status and Type, National Health and Nutrition Examination Survey, 2001-2010
}

\author{
Alissa Stevens, MPH; Elizabeth Courtney-Long, MA, MSPH; Cathleen Gillespie, MS; \\ Brian S. Armour, PhD
}

\begin{abstract}
Suggested citation for this article: Stevens A, Courtney-Long E, Gillespie C, Armour BS. Hypertension Among US Adults by Disability Status and Type, National Health and Nutrition Examination Survey, 2001-2010. Prev Chronic Dis 2014; 11:140162. DOI: http://dx.doi.org/10.5888/pcd11.140162.
\end{abstract}

\section{PEER REVIEWED}

\section{Abstract}

The prevalence of hypertension among people with disabilities is not well understood. We combined data from the 2001-2010 National Health and Nutrition Examination Survey to obtain estimates of hypertension prevalence by disability status and type (cognitive, hearing, vision, or mobility limitation) and assess the association between disability and hypertension. Overall, $34 \%$ of adults with disabilities had hypertension compared with $27 \%$ of adults without disabilities; adults with mobility limitations were more likely to have hypertension than adults without disabilities (adjusted prevalence ratio: 1.23; 95\% confidence interval: 1.16-1.32). Our results suggest that adults living with disabilities are an important subpopulation to include in hypertension reporting and intervention efforts.

\section{Objective}

Hypertension is a key treatable risk factor for cardiovascular disease, a leading cause of illness and death (1). It affects approximately $30 \%$ of US adults (2). An Institute of Medicine (IOM) report states that increasing analysis and reporting on understudied subpopulations can improve awareness, treatment, and control of hypertension (3).
Certain subpopulations, such as racial and ethnic minorities, people of low socioeconomic status, and older people are at high risk for hypertension $(3,4)$. More than 56 million people in the United States have a disability (5) and experience health disparities (6), yet hypertension among this group has not been well researched. Our objectives were to estimate hypertension prevalence by disability status and type and assess the association between disability and hypertension using nationally representative data.

\section{Methods}

We used data from the 2001-2010 National Health and Nutrition Examination Survey (NHANES). NHANES is a multistage probability sample of the US civilian noninstitutionalized population that combines interview data with clinical information obtained from physical examinations to assess the population's health and nutritional status (7). We limited our analyses to participants aged 20 years or older and excluded pregnant women $(n=1,011)$ and participants who had missing information on either disability $(\mathrm{n}=$ 221) or all 3 blood pressure measurements or hypertension medication use $(n=2,690)$, yielding a final sample size of 23,800 .

We defined hypertension as an average systolic blood pressure of $140 \mathrm{~mm} \mathrm{Hg}$ or higher or an average diastolic blood pressure of 90 $\mathrm{mm} \mathrm{Hg}$ or higher, based on up to 3 blood pressure measurements, or participant report of taking blood pressure-lowering medication. We defined disability based on basic actions difficulty (8) as a limitation in 1 or more of the following domains: cognitive (difficulty remembering or experiencing periods of confusion), hearing (moderate or a lot of trouble hearing, or deaf), vision (trouble seeing even when wearing glasses or contact lenses), or mobility (some or much difficulty or unable to walk for one-quarter of a mile; walk up 10 steps; stoop, crouch, or kneel; lift or carry something as heavy as 10 pounds; walk between rooms on the 
same level; stand up from an armless chair; stand for about 2 hours; sit for about 2 hours; reach up over head; or grasp or handle small objects).

We used SAS-callable SUDAAN, version 11.0 (9) to account for the complex survey design. Data were weighted using examination sample weights. We calculated age-adjusted prevalence estimates of hypertension by disability status and type. We used logistic regression to obtain prevalence ratios for the association between disability status and type and hypertension adjusted for age, sex, race/ethnicity, education level, income-to-poverty ratio (calculated by dividing family income by the federal poverty level specific to family size, year, and state), health insurance status, and times received care in the previous 12 months.

\section{Results}

Prevalence of hypertension among US adults aged 20 or older was $30.0 \%$ (95\% confidence interval [CI], 29.2\%-30.8\%). The prevalence of disability overall was $37.9 \%$ (95\% CI, 36.9\%-38.9\%). Prevalence of disability types ranged from $6.3 \%$ (95\% CI, $5.9 \%-6.8 \%$ ) for cognitive limitation to $25.6 \%$ (95\% CI, $24.7 \%-26.5 \%$ ) for mobility limitation. Respondents were primarily aged 20 to 44 years of age (48.0\%), were non-Hispanic white (71.2\%), had at least a high school education $(81.1 \%)$, and had some type of health insurance ( $80.9 \%$ ) (Table 1).

Age-adjusted prevalence of hypertension was significantly higher among respondents with disabilities than among those without $(34.2 \%$ vs $26.9 \% ; P<.001)$. By disability type, the prevalence of hypertension ranged from $29.7 \%(95 \%$ CI, 27.0-32.5) among adults with hearing limitation to $39.1 \%$ (95\% CI, 37.4\%-40.8\%) among adults with mobility limitation (Table 2). With the exception of adults with hearing limitation, the prevalence of hypertension was significantly higher among adults with each disability type than among adults without any disability.

After controlling for sociodemographic variables, adults with disabilities had a $13 \%$ higher prevalence of hypertension than adults without any disability (adjusted prevalence ratio $[\mathrm{APR}]=1.13$; 95\% CI, 1.08-1.20). There were significantly higher prevalence ratios for adults with most types of disability than for adults without any disability, the highest being for adults with mobility limitation (APR $=1.23 ; 95 \%$ CI, 1.16-1.32) and the lowest for adults with vision limitation (APR $=1.12 ; 95 \% \mathrm{CI}, 1.04-1.20$ ). There was no significant difference after adjusting for sociodemographic variables in the prevalence of hypertension between adults with hearing limitation and adults without disability (Table 2).

\section{Discussion}

We found that adults with disabilities are more likely to have hypertension, even after controlling for sociodemographic and health care access indicators. This disparity, and the variation by disability type, may in part be explained by behavioral risk factors such as obesity, smoking, and physical inactivity that co-occur with or worsen the effects of hypertension $(1,10)$ and that disproportionately affect people with disabilities $(6,11)$. For example, the prevalence of movement difficulty is 1.5 to 2 times higher among obese adults than among adults with a normal body mass index (12).

There are several limitations to our analysis. First, NHANES data are limited to the community-dwelling population and exclude people who reside in institutional settings. This exclusion may result in an underestimate of hypertension prevalence among adults with a disability. Second, the data collection methods are crosssectional and do not allow us to draw inferences about the causality between disability and hypertension. Third, blood pressure was measured during a single visit, which may overestimate or underestimate hypertension prevalence (1). However, this factor would likely affect people with and without disabilities in a similar manner and should not have affected the magnitude of the disparities noted here.

Citing the public health significance of hypertension, a recent IOM report noted the importance of identifying at-risk subpopulations (3). To our knowledge this is the first study to describe hypertension by disability status and type in a nationally representative US sample. Consistent with recent studies among the general population, we controlled for sociodemographic and health care access indicators (2). Future studies should explore behavioral factors that could also be related and differences in treatment and control of hypertension for adults with disabilities. Our results suggest that adults living with disabilities are an important subpopulation to include in reporting and intervention efforts that work toward reducing hypertension prevalence.

\section{Acknowledgments}

This research received no specific grant from any funding agency in the public, commercial, or nonprofit sectors.

\section{Author Information}

Corresponding Author: Alissa Stevens, MPH, Division of Human Development and Disability, Centers for Disease Control and Prevention, 1600 Clifton Rd NE, Mail Stop E-88, Atlanta, GA 30333. Telephone: 404-498-2606. E-mail: astevens@cdc.gov.

\footnotetext{
The opinions expressed by authors contributing to this journal do not necessarily reflect the opinions of the U.S. Department of Health and Human Services, the Public Health Service, the Centers for Disease Control and Prevention, or the authors' affiliated institutions.
} 
Author Affiliations: Elizabeth Courtney-Long, Brian S. Armour, Division of Human Development and Disability, Centers for Disease Control and Prevention, Atlanta, Georgia; Cathleen Gillespie, Division for Heart Disease and Stroke Prevention, Centers for Disease Control and Prevention, Atlanta, Georgia.

\section{References}

1. Chobanian AV, Bakris GL, Black HR, Cushman WC, Green LA, Izzo JLJr, et al. Seventh report of the Joint National Committee on Prevention, Detection, Evaluation, and Treatment of High Blood Pressure. Hypertension 2003; 42(6):1206-52.

2. Centers for Disease Control and Prevention. Vital signs: awareness and treatment of uncontrolled hypertension among adults - United States, 2003-2010. MMWR Morb Mortal Wkly Rep 2012;61(35):703-9.

3. Institute of Medicine. A population-based policy and systems change approach to prevent and control hypertension. Washington (DC): The National Academies Press; 2010.

4. Schneider RH, Staggers F, Alexander CN, Sheppard W, Rainforth M, Kondwani K, et al. A randomized controlled trial of stress reduction for hypertension in older African Americans. Hypertension 1995;26(5):820-7.

5. Brault MW. Americans with disabilities: 2010. Current Population Reports, P70-131. Washington (DC): US Census Bureau; 2012.

6. Pharr JR, Bungum T. Health disparities experienced by people with disabilities in the United States: a Behavioral Risk Factor Surveillance System study. Glob J Health Sci 2012; 4(6):99-108.

7. National Health and Nutrition Examination Survey. Questionnaires, datasets, and related documentation. Centers for Disease Control and Prevention, National Center for Health Statistics; 2001-2010. http://www.cdc.gov/nchs/nhanes.htm. Accessed May 29, 2012.

8. Altman B, Bernstein A. Disability and health in the United States, 2001-2005. Hyattsville (MD): National Center for Health Statistics; 2008.

9. SUDAAN release 11.0. Research Triangle Park (NC): RTI International; 2012.

10. US Department of Health and Human Services. How tobacco smoke causes disease: the biology and behavioral basis for smoking-attributable disease: a report of the Surgeon General. Atlanta (GA): Centers for Disease Control and Prevention, National Center for Chronic Disease Prevention and Health Promotion, Office on Smoking and Health; 2010.
11. Centers for Disease Control and Prevention. Vital signs: disability and physical activity — United States, 2009-2012. MMWR Morb Mortal Wkly Rep 2014;63(18):407-13.

12. Armour BS, Courtney-Long E, Campbell VA, Wethington HR. Estimating disability prevalence among adults by body mass index: 2003-2009 National Health Interview Survey. Prev Chronic Dis 2012;9:E178 http://www.cdc.gov/pcd/issues/2012/ 12_0136.htm Accessed June 28, 2013.

The opinions expressed by authors contributing to this journal do not necessarily reflect the opinions of the U.S. Department of Health and Human Services, the Public Health Service, the Centers for Disease Control and Prevention, or the authors' affiliated institutions. 


\section{Tables}

Table 1. Study of Relationship Between Hypertension and Disability Type: Characteristics of Participants $(\mathrm{N}=23,800)$, National Health and Nutrition Examination Survey, 2001-2010

\begin{tabular}{|c|c|c|}
\hline Characteristic & Unweighted $\mathrm{n}^{\mathrm{b}}$ & Weighted \% (95\% Cl) \\
\hline Hypertension & 8,900 & $30.0(29.2-30.8)$ \\
\hline \multicolumn{3}{|l|}{ Disability } \\
\hline Any disabilityc & 10,766 & $37.9(36.9-38.9)$ \\
\hline Cognitive limitation & 2,008 & $6.3(5.9-6.8)$ \\
\hline Hearing limitation & 1,937 & $6.6(6.3-7.0)$ \\
\hline Vision limitation & 4,918 & $17.7(17.0-18.5)$ \\
\hline Mobility limitation & 7,851 & $25.6(24.7-26.5)$ \\
\hline \multicolumn{3}{|l|}{ Age, $y^{d}$} \\
\hline $20-44$ & 9,895 & $48.0(46.6-49.4)$ \\
\hline $45-64$ & 7,727 & $35.1(34.0-36.2)$ \\
\hline$\geq 65$ & 6,178 & $16.9(16.0-17.8)$ \\
\hline \multicolumn{3}{|l|}{ Sex } \\
\hline Male & 11,981 & $49.2(48.6-49.7)$ \\
\hline Female & 11,819 & $50.9(50.3-51.4)$ \\
\hline \multicolumn{3}{|l|}{ Race/ethnicitye } \\
\hline Non-Hispanic white & 12,009 & $71.2(68.4-73.8)$ \\
\hline Non-Hispanic black & 4,720 & $10.9(9.6-12.5)$ \\
\hline Mexican American & 4,494 & $8.0(6.7-9.5)$ \\
\hline \multicolumn{3}{|l|}{ Education } \\
\hline Less than high school & 6,945 & $18.9(17.7-20.0)$ \\
\hline High school graduate/GED & 5,749 & $25.2(24.3-26.2)$ \\
\hline Some college/associate's degree & 6,463 & $30.4(29.5-31.4)$ \\
\hline College graduate or higher & 4,611 & $25.5(23.9-27.1)$ \\
\hline \multicolumn{3}{|l|}{ Income-to-poverty ratiof } \\
\hline$<1.00$ & 4,176 & $13.1(12.3-14.1)$ \\
\hline $1.00-1.99$ & 5,851 & $20.6(19.6-21.6)$ \\
\hline $2.00-4.99$ & 8,072 & $41.6(40.4-42.9)$ \\
\hline
\end{tabular}

Abbreviation: $\mathrm{Cl}$, confidence interval; GED, general educational development.

a Age-adjusted to the 2000 US standard population by direct method using age groups 20-44, 45-64, and $\geq 65$.

b Some categories do not sum to 23,800 due to missing responses.

c Disability types are not mutually exclusive and unweighted sample sizes will not sum to the total for "Any disability."

${ }^{\mathrm{d}}$ Not age-adjusted.

e Due to changes in oversampling rates across survey cycles, estimates for Hispanics could not be produced; race/ethnicity was categorized as non-Hispanic white, non-Hispanic black, and Mexican American. Data on respondents reporting "other" race/ethnicity are not presented but are included in total estimates.

${ }^{f}$ Calculated by dividing family income by the federal poverty level specific to family size, year, and state.

(continued on next page)

The opinions expressed by authors contributing to this journal do not necessarily reflect the opinions of the U.S. Department of Health and Human Services, the Public Health Service, the Centers for Disease Control and Prevention, or the authors' affiliated institutions. 
(continued)

Table 1. Study of Relationship Between Hypertension and Disability Type: Characteristics of Participants $(\mathrm{N}=23,800)$, National Health and Nutrition Examination Survey, 2001-2010

\begin{tabular}{|c|c|c|}
\hline Characteristic & Unweighted $\mathrm{n}^{\mathrm{b}}$ & Weighted \% (95\% Cl) \\
\hline$\geq 5.00$ & 3,960 & $24.7(23.2-26.2)$ \\
\hline \multicolumn{3}{|c|}{ Health insurance } \\
\hline Medicare & 6,265 & $18.6(18.2-19.0)$ \\
\hline Private & 10,049 & $55.0(53.7-56.3)$ \\
\hline Public & 2,033 & $7.3(6.8-7.8)$ \\
\hline Uninsured & 5,272 & $19.2(18.2-20.2)$ \\
\hline \multicolumn{3}{|c|}{ Times received care in past 12 months } \\
\hline 0 or 1 & 8,218 & $36.0(35.1-36.8)$ \\
\hline 2 or 3 & 6,186 & $27.1(26.4-27.9)$ \\
\hline 4 or more & 9,381 & $37.0(36.1-37.8)$ \\
\hline
\end{tabular}

Abbreviation: $\mathrm{Cl}$, confidence interval; GED, general educational development.

${ }^{\text {a }}$ Age-adjusted to the 2000 US standard population by direct method using age groups $20-44,45-64$, and $\geq 65$.

b Some categories do not sum to 23,800 due to missing responses.

c Disability types are not mutually exclusive and unweighted sample sizes will not sum to the total for "Any disability."

d Not age-adjusted.

e Due to changes in oversampling rates across survey cycles, estimates for Hispanics could not be produced; race/ethnicity was categorized as non-Hispanic white, non-Hispanic black, and Mexican American. Data on respondents reporting "other" race/ethnicity are not presented but are included in total estimates.

${ }^{f}$ Calculated by dividing family income by the federal poverty level specific to family size, year, and state. 
Table 2. Age-Adjusted ${ }^{a}$ Prevalence of Hypertension and Adjusted Prevalence Ratios ${ }^{b}$ of Hypertension, by Disability Status and Type, Adults Aged 20 Years or Older, National Health and Nutrition Examination Survey, 2001-2010

\begin{tabular}{|l|r|r|}
\hline Disability Status & \% Hypertension $(95 \% \mathrm{Cl})$ & \multicolumn{1}{|c|}{ Hypertension APR (95\% Cl) } \\
\hline Any disabilityc & $34.2(33.1-35.4)^{\mathrm{d}}$ & $1.13(1.08-1.20)$ \\
\hline Cognitive limitation & $36.4(33.8-39.1)^{\mathrm{d}}$ & $1.16(1.05-1.28)$ \\
\hline Hearing limitation & $29.7(27.0-32.5)$ & $0.99(0.91-1.09)$ \\
\hline Vision limitation & $32.9(31.5-34.4)^{\mathrm{d}}$ & $1.12(1.04-1.20)$ \\
\hline Mobility limitation & $39.1(37.4-40.8)^{\mathrm{d}}$ & $1.23(1.16-1.32)$ \\
\hline No disability & $26.9(25.8-28.1)$ & $1[$ Reference] \\
\hline
\end{tabular}

Abbreviation: $\mathrm{Cl}$, confidence interval; APR, adjusted prevalence ratio.

a Age-adjusted to the 2000 US standard population by direct method using age groups 20-44, 45-64, and $\geq 65$.

${ }^{\mathrm{b}}$ Adjusted for age, sex, race/ethnicity, education level, income-to-poverty ratio, health insurance status, and times received care in the previous 12 months.

${ }^{\mathrm{C}}$ Disability types are not mutually exclusive.

d $P<.001$ when compared with "No disability"; $P$ values determined using a 2-tailed $t$ test.

\footnotetext{
The opinions expressed by authors contributing to this journal do not necessarily reflect the opinions of the U.S. Department of Health and Human Services, the Public Health Service, the Centers for Disease Control and Prevention, or the authors' affiliated institutions.
} 